\title{
Evolution of grinding energy and particle size during dry ball-milling of silica sand
}

\author{
Nicolas Blanc ${ }^{\mathrm{a}}$, Claire Mayer-Laigle ${ }^{\mathrm{a}}$, Xavier Frank ${ }^{\mathrm{a}}$, Farhang Radjai ${ }^{\mathrm{b}}$, Jean-Yves Delenne ${ }^{\mathrm{a},{ }^{*}}$ \\ ${ }^{a}$ IATE, Univ Montpellier, CIRAD, INRAE, Montpellier SupAgro, Montpellier, France \\ ${ }^{b}$ LMGC, Univ Montpellier, CNRS, Montpellier, France
}

\begin{abstract}
The energy efficiency of dry fine grinding process of powders dramatically declines with decreasing particle size, and represents a growing concern in various cutting-edge technical applications for pharmaceutical and cosmetic products, advanced-ceramics, and food industry. In this paper, we report on a detailed experimental analysis of the long-term grinding behavior of silica sand as a model material in an oscillatory ball mill for a broad range of the values of vibration frequency and amount of powder. We show that the re-agglomeration of fine particles unambiguously explains the anomalous increase of grinding power consumption. We also find that the agglomeration phenomena are correlated with the volume fraction of the class of finest particles. A nonlinear comminution model is shown to capture this effect for the prediction of the evolution of the specific surface area.

Keywords: Milling, agglomeration, Rittinger law, powder, grinding limit

2020 MSC: 00-01 99-00
\end{abstract}

\section{Introduction}

The grinding process is used extensively for the manufacture of cement, pharmaceutical and food products with various purposes such as calibrating the size of a raw product, extracting specific components or increasing the specific surface area and the reactivity of products. It is well known that the industrial grinding operations represent a major part of the total energy consumption at a global level. For example, clinker grinding consumes one third of the total energy needed for cement manufacturing [1]. Therefore, improving our understanding of the mechanisms underlying energy dissipation in milling operations has remained as a major challenge for the industry with

*jean-yves.delenne@inrae.fr 
the aim of reducing their ecological footprint.

The energy consumption is affected by not only the properties and mechanical behavior of the material and the grinder technology but also the loading mode and the rate of energy supply. The grinding device controls the way energy is supplied to the material and the dissipation mechanisms involved in the process such as friction and the viscosity of the carrier fluids. The material can be more or less brittle, dry or dense. It evolves during the grinding process with an increasing cohesive stress, defined as the additional stress induced by cohesive forces between particles, as the average particle diameter is reduced. Hence, for fine-milling, both inter-particle and intra-particle surface energies are to be overcome for grinding the material [2]. In all cases, the grinding efficiency is very low. According to Schönert (cited by Tavares and King [3]) only $4 \%$ to $8 \%$ of the total energy supplied is really consumed for the creation of new surfaces.

Several comminution laws have been proposed to link the consumed energy to a characteristic particle size of the powder. The popular models are those of Rittinger [4], Bond [5] and Kick [6]. While the Bond and Kick models are mostly employed for coarse and intermediate size grinding, the Rittinger model is better suited for fine comminution [7]. This model assumes a linear relation between the specific surface area per unit of mass $S_{m}$ of the powder and the energy per unit of mass $E_{m}$ :

$$
\frac{d S_{m}}{d E_{m}}=\tau,
$$

where $\tau$ is a constant parameter governing the kinetic process. As the evolution of the system is controlled by the energy power input (which is generally constant), $\tau$ represents the rate of production of specific surface area. Note that an alternative writing of this model is frequently employed using the median particle size $d_{50} \simeq 1 / S_{m}$ and assuming spherical particles.

Chen et al. [8] showed for an oscillatory ball mill that the median particle diameter $d_{50}$ (and also other percentiles such as $\mathrm{d} 10$ and $\mathrm{d} 90$ ) were well fitted by Rittinger's model for the grinding of $\alpha$-Lactose from 450 to $150 \mu \mathrm{m}$. For experiments on spruce saw dust on the same type of mill, Karinkanta et al. [9] showed, however, that $d_{50}$ tends to deviate substantially from the linear trend for very fine milling $(<20 \mu \mathrm{m})$. This observation was attributed to the existence of a limit size in fracturing particles. For example, for dry silica sand milling a limit size of $1 \mu \mathrm{m}$ was observed by Mucsi [7]. Cho et al. [10] found a limit size of $30 \mathrm{~nm}$ for silica sand in a stirred-media 
grinder where the particles were in suspension in water. The presence of water shields the inter-particle adhesion and hence it should decrease the formation of agglomerates that appear in fine dry milling [11]. Other possible effects which reduce the grindability are: i) the existence of a brittle to ductile transition that slows down the cracks propagation in particles [12,13], ii) confined particles under high confinement have higher compression strength [14], iii) due to local interactions, a coating of grinding balls by the powder may occur, reducing thus stress transmission [15], iv) fine particles enhance air trapping inside the bed, leading to cushioning effects [15]

To account for this limit size, Kano et al. fitted the variation of $d_{50}$ with a saturating exponential law [16]. This type of limit was also introduced in balance models [15, 17] or directly in comminution law such as in the Tanaka model [2], which modifies the Rittinger model by assuming a limit surface $[18,19,20]$.

In this paper, we present a detailed investigation of the dry grinding of silica sand in an oscillatory ball mill. We are interested in the evolution of specific surface area (SSA), particle size distribution, agglomerated SSA and consumed electrical energy as a function of the input grinding energy power. These measurements allow us to characterize the onset of agglomeration and its signature on the evolution of SSA as a deviation from the linear Rittinger regime and its level-off at larger input power. We also evidence the correlation of particle agglomeration with the production of very fine particles whose volume fraction increases proportionally to the SSA. We introduce a phenomenological model that accounts for the agglomeration by an extra energy dissipation term proportional to the created surface. The predicted asymptotic SSA is in agreement with previous experimental measurements in a ball mill.

\section{Materials and Methods}

\subsection{Raw material}

In this work, we used a commercial silica sand composed of $98.60 \%$ of pure silica for filtration applications, marketed by Euromortier. It is a sieved sand between $0.5 \mathrm{~mm}$ and $1.4 \mathrm{~mm}$. All the samples used in this study come from the same batch. Silica sand is employed in many industrial applications and its brittle mechanical behavior is well described in the literature. Furthermore, silica particles present the advantage of being relatively homogeneous, inert, safe and easy to 
handle, making it a good model material. Silica sand is a relatively hard material. It can scratch glass and steel and it has an index of 7 on the qualitative Mohs scale. Using a helium gas pycnometer (Ultrapyc 1200e, Quantachrome Instruments ${ }^{\circledR}$ ) the density was measured and found to be very close to that of $\alpha$-quartz $\left(\rho_{\alpha \text {-quartz }} \simeq 2.65\right.$, implying that the particles are mostly crystalline and not amorphous $\left(\rho_{\text {amorphous }} \simeq 2.20\right.$ ).

The commercial silica sand was sieved using a Rotex sieving machine to ensure that it was properly graded and to reduce spreading so as to achieve a nearly monodisperse population. We chose two sieves with close aperture diameters $0.8 \mathrm{~mm}$ and $1.0 \mathrm{~mm}$ and with a small size allowing us to do measurements using laser diffraction from the very beginning of grinding. After $10 \mathrm{~min}$ sieving, we obtained a fraction representing $10 \%$ of the total initial mass. The bulk density was measured to be between 1.4 and $1.6 \mathrm{~g} . \mathrm{cm}^{-3}$. The sieved material was used as input raw material in all experiments reported in this paper.

\subsection{Milling device and grinding energy}

Fig. 1a shows the oscillatory ball mill (Retsch ${ }^{\circledR}$ MM400) used in this study and a scheme (Fig. 1b) representing one of its two $50 \mathrm{~mL}$ milling jars. Each jar is initially filled with a mass $M$ of raw material and a single $25 \mathrm{~mm}$-diameter steel ball. The jars vibrate horizontally at a frequency chosen between 3 to $30 \mathrm{~Hz}$. The motion of the jar follows a quasi-straight line with a periodic signal:

$$
x_{j}(t)=A \sin (2 \pi f t)
$$

where $f$ is the vibration frequency and $t$ is time. The stroke amplitude of the jar was $A=7.2$ $\mathrm{mm} \pm 50 \mu \mathrm{m}$ independently of frequency. It was measured by means of a contactless position sensor.

Figure 1: a) Picture of the ball mill MM400 used in experiments. b) Scheme of the grinding jar.

The electric power supplied to the device sets in motion the ball and the powder that it contains. The kinetic energy supplied in this way is consumed in the grinding process, but a large amount of power is dissipated by friction between the different mechanical parts of the device and through Joule effect in wires. It is therefore difficult to estimate the grinding energy, i.e. the energy 
for the comminution of particles. We may consider that grinding energy is directly related to the ball-jar collision energy. Following the work of Karinkanta et al [9], the friction between the ball and the jar is assumed to be negligible compared to the impact energy.

The maximum velocity of the jar, according to Eq. 2, is

$$
v_{j}^{\max }=2 A \pi f
$$

Since the ball is propelled by motion of the jar, its maximum velocity $v_{b}^{\max }$ is equal to that of the jar $v_{j}^{\max }$. We assume that the ball-jar impact occurs when the ball and the jar have the same velocity $v_{j}^{\max }$ in opposite directions. This corresponds to a maximal impact energy $E_{i}=\frac{1}{2} m_{b}\left(v_{j}^{\max }+v_{b}^{\max }\right)^{2}=8 m_{b}(A \pi f)^{2}$. The specific grinding energy $E_{m}$ is the specific impact energy $E i_{m}=E i / M$ multiplied by the number of impacts $N_{i}=f t$ during the grinding time $t$. Hence,

$$
E_{m}(t)=\frac{E i}{M} N_{i}=\frac{16 m_{b}(A \pi)^{2} f^{3} t}{M}
$$

Note that the assumptions underlying this relation hold at weak filling ratio where the motion of the ball is not limited by the amount of material in the jar.

\subsection{Parametric study}

We performed a detailed parametric study in which the energy was changed by varying the frequency, the mass of the powder (related to the filling ratio) and the milling time. For the chosen range of frequencies, the ball has a high kinetic energy and the mechanical work is mostly transmitted through impacts of the ball onto the powder. Using a transparent jar, Chen et al. [8] have shown that for frequencies below $15 \mathrm{~Hz}$ the main loading mode is the rolling of the ball. Therefore, we considered three frequencies above $15 \mathrm{~Hz}$ and up to $25 \mathrm{~Hz}$ with four different values of silica sand sample mass ranging from 6 to $20 \mathrm{~g}$. Note that increasing the mass of product (thus the filling ratio) reduces the stroke of the ball.

All the samples were first dried in an oven at $65{ }^{\circ} \mathrm{C}$ during 24 hours. A first series of experiments were conducted at $20 \mathrm{~Hz}$ for different values of powder mass $(6,13,17$ and $20 \mathrm{~g})$ and a second series were then conducted with a fixed mass (13 g) for two other frequencies (15 and 25 $\mathrm{Hz}$ ). For each set of parameters, 10 independent grinding experiments were conducted for times 
ranging from 0.5 to $111 \mathrm{~min}$.

For all these experiments, the consumed electric power $P$ by the device was measured with a wattmeter Meteix ${ }^{\circledR}$ PX 120 at the level of the electric grid. A laser thermometer Optris ${ }^{\circledR}$ CT LT20 was also employed to monitor the jar surface temperature during the grinding at the center of the jar.

\subsection{Characterization of the ground powder}

At the scale of particles, scanning electron microscope (SEM) imaging was performed. At the scale of the bulk, the particle size distribution and specific surface area (SSA) was also determined.

\subsubsection{Scanning electron microscopy}

Observations of different samples of the ground powder were performed using a Phenom Pro-X SEM (France) equipped with a backscattered electron detector. The particles were directly fixed without coating on the sample holder using carbon adhesive tape and the images were captured at a voltage of $15 \mathrm{kV}$.

\subsubsection{Particle size distribution}

The particle size distribution was determined by Laser Diffraction (LD) using a Mastersizer 2000 (Malvern Instruments Ltd., United Kingdom) particle size analyzer coupled with the sample dispersion unit Hydro 2000S with ethanol as liquid carrier. Note that, as a large number of the studies carried out in our laboratory involve water-reactive powders, we preferred to use ethanol rather than water also for these experiments although water could have been equivalently used. The results were quantified in volume based on the Mie theory [21]. From this particle size distribution, the SSA per unit of mass denoted $S_{L D}$ (in kg. $\mathrm{m}^{-2}$ ) was evaluated as

$$
S_{L D}=\frac{1}{\rho} \sum_{i} \frac{3 \alpha_{i}}{R_{i}},
$$

where $\rho$ is the density of silica, $i$ the index grading class (between 0.02 and $2000 \mu \mathrm{m}$ ), $\alpha_{i}$ is the volume fraction and $R_{i}$ the average radius of particles inside the size class $i$. For each sample, 3 measurements were done, and the standard deviation of the measurements were calculated. 
To estimate the level of agglomeration in the bulk, we used ultrasounds with the following protocol:

- A first measurement is directly done on the powder (denoted $S_{L D}^{\star}$ ).

- 3 minutes of ultrasonic treatment is applied to the suspension.

- The sample is stirred at $2000 \mathrm{rpm}$ during 5 minutes in order to eliminate potential bubbles.

- A second measurement is performed $\left(S_{L D}\right)$.

The agglomerated surface of the powder is calculated as

$$
S^{a g l}=S_{L D}-S_{L D}^{\star}
$$

\subsubsection{Measurement of the BET surface}

The Brunauer-Emmett-Teller (BET) method, based on nitrogen absorption, was used as a reference measurement of the SSA. This measure, denoted below $S_{B E T}$, is expressed in units of mass as in the case of $S_{L D}$. We used a ASAP 2460 (Micrometrics ${ }^{\circledR}$ ) with a liquid nitrogen cooling system. Prior to measurements, the samples were degassed at $250^{\circ} \mathrm{C}$ during 48 hours under vacuum using a VacPrep 061 (Micrometrics ${ }^{\circledR}$ ). Although this method provides an accurate value of the SSA, it has the disadvantage of being time-consuming and expensive (48 hours per sample for BET against $15 \mathrm{~min}$ with laser granulometry). For this reason, only the $20 \mathrm{~Hz}$ milled samples were tested by this method and used to calibrate the measurements by laser granulometer for all the tests with other values of parameters.

\section{Results and Discussions}

\subsection{Particle Size distribution and specific surface area}

Fig. 2 shows an example of the evolution of particle size distribution with time for a $13 \mathrm{~g}$ sample milled at $20 \mathrm{~Hz}$. We see that, although the powder used as input was first calibrated between 0.8 and $1.0 \mathrm{~mm}$, after $1.5 \mathrm{~min}$ of milling the particles are already broadly distributed over nearly 3 orders of magnitude. While milling, the main peak of the distribution shift from $100 \mu \mathrm{m}$ to 2 $\mu \mathrm{m}$.Fig. 3 shows SEM images of the powder for two values of milling duration. As evidenced by the PSD, the particles are highly polydisperse with angular polyhedral shapes. At the beginning of milling, the particles are slightly elongated with an aspect ratio between 2 and 3 (Fig. 3a). This 
elongation tends to decrease with time (Fig. 3b).

Figure 2: Evolution of PSD with time (from 1.5 to $65 \mathrm{~min}$ ) for $13 \mathrm{~g}$ of silica sand milled at $20 \mathrm{~Hz}$. The feed size distribution is represented by the hatched area.

Figure 3: SEM images of silica sand particles at short milling (6 g, $20 \mathrm{~Hz})$ duration (30 s) (a) and at the end of the milling (29 min) (b). An example of agglomerates appearing due to cohesive interactions with finest particles at long milling time is also shown (c).

Figure 4: Calibration curve $S_{B E T}$ as a function of $S_{L D}$ for $13 \mathrm{~g}$ of silica sand milled at $20 \mathrm{~Hz}$.

Fig. 4 shows the SSA $S_{B E T}$ measured by BET as a function of $S_{L D}$ determined by using the laser diffraction method (based on the assumption of spherical shapes for particles). The data are well-fitted by a power law:

$$
S_{B E T}=0.0258\left(S_{L D}\right)^{1.739}
$$

For low surface values the ratio $S_{B E T} / S_{L D} \simeq 1.5$ and increases up to $\simeq 3.7$ for $S_{L D}=900$. This difference can be attributed to the agglomeration of small particles as shown in Fig. 3c. In fact, as nitrogen molecules used in BET measurements are very small, they penetrate into the pore space inside the agglomerated particles. On the contrary, in laser diffraction an agglomerate is seen as a coarse particle.

As $S_{B E T}$ measurements are closer to the real specific surface, all the measurements were scaled using the calibration curve of Fig. 4. To simplify notations, we will use $S$ for the SSA evaluated according to this method.

\subsection{Effect of milling conditions}

In Fig. 5 all the data are plotted as a function of time. We clearly distinguish two parts on these curves: a nearly linear rapid increase at the beginning, followed by level-off at an upper limit of $S=S_{\infty}$ around $3200 \mathrm{~m}^{2} \mathrm{~kg}^{-1}$. As expected, the initial slope depends in a systematic way on the frequency and the mass of the product. We see that the milling rate increases both with the number of impacts (related to the frequency) and with the decrease of the amount of powder. 
Figure 5: Evolution of the SSA of the powder as a function of time.

The same data are plotted in Fig 6 as a function of the specific milling energy given by Eq. 4. Interestingly, all our data points quite nicely collapse on a master curve independently of the values of filling ratio and frequency. This collapse indicates that for the range of parameters considered in this work the extra surface created by milling fully depends on the total energy supplied. Hence, in the investigated range of impact energies, the parameter $E_{m}$ is the control parameter of the generated SSA and mediates the influence of all experimental parameters on the grinding process. Note that, for the considered range of values of experimental parameters, $E_{i}$ varies by a factor 3 and $E i_{m}$ by a factor 4 .

Figure 6: Evolution of the SSA as a function of the specific grinding energy for all experimental data. The Rittinger model (black dotted line) and a modified Rittinger model (red solid line) are shown; see text.

The Rittinger model, which assumes a linear increase of the surface as a function of the grinding energy (Eq. 1), is plotted in the same figure. This model is consistent with the experimental data up to a surface of approximately $1500 \mathrm{~m}^{2} \mathrm{~kg}^{-1}$. Beyond this value, the increase of $S$ is more energy-consuming than the linear model. An additional amount of energy is required to overcome the dissipation due to several different effects. One important effect is the increase of yield stress with decreasing particle size [22], a well-known scale effect due to a decreasing probability of finding flaws above the critical size for the onset of crack propagation according to the Griffith criterion $[23,24]$. In this respect, it is also expected that an increasing amount of energy is dissipated by plastic deformations of the particle [13, 25]. Another important effect is that finer particles produced by grinding form a cushion between larger particles, thereby reducing stress concentration and thus the probability of particle fracture (cushioning effect) [15]. Finally, a major effect is the increase of cohesive stresses induced by surface forces in the granular bed as mean particle size declines. For micron-size particles, this internal cohesion prevails as compared with confining stresses and gravity $[2,26,27,28]$. For this reason, an increasing amount 
of the grinding energy is consumed to overcome cohesive forces between particles or break the agglomerates of fine particles that form spontaneously inside the powder bed.

\subsection{Modified Rittinger Model}

The nonlinear evolution of the SSA can be accounted for by assuming an extra energy dissipation that is proportional to the created surface $S$. The Rittinger model can thus be modified as

$$
\frac{d S}{d E_{m}}=\tau-\gamma \frac{S}{S_{0}}
$$

where $S_{0}\left(\mathrm{~m}^{2} \mathrm{~kg}^{-1}\right)$ is the initial SSA of the input powder, $\tau$ is the maximum comminution rate corresponding to the created surface per unit of energy (expressed in $\mathrm{m}^{2} \mathrm{MJ}^{-1}$ ) and $\gamma$ (in $\mathrm{m}^{2} \mathrm{MJ}^{-1}$ ) is a phenomenological parameter accounting for all the dissipative effects briefly mentioned previously.

Eq. (8) leads to an asymptotic behavior where $d S / d E_{m}=0$ and $S=S_{\infty}=\tau S_{0} / \gamma$. Replacing $\gamma$ by $\tau S_{0} / S_{\infty}$ in Eq. (8) leads to the following equation:

$$
\frac{d S}{d E_{m}}=\tau\left(1-\frac{S}{S_{\infty}}\right) .
$$

This equation is similar to that introduced by Tanaka in $1954[2,18]$ with a slightly different normalization.

The experimental data are well fitted ( $R^{2}=0.99$ with the least squared method) by the integrated form of Eq. 8:

$$
S\left(E_{m}\right)=S_{0} \frac{\tau}{\gamma}\left[1-\left(1-\frac{\gamma}{\tau}\right) \exp \left(-\frac{\gamma E_{m}}{S_{0}}\right)\right]
$$

where $S_{0}=31.4 \mathrm{~m}^{2} \mathrm{~kg}^{-1}, \tau=8980 \mathrm{~m}^{2} \mathrm{MJ}^{-1}$ and $\gamma=89.8 \mathrm{~m}^{2} . \mathrm{MJ}^{-1}$. These values of the parameters are consistent with the measured value of $S_{\infty} \simeq 3140 \simeq \tau S_{0} / \gamma \mathrm{m}^{2} \mathrm{~kg}^{-1}$. The value of $\tau$ is in good agreement (difference below 4\%) with the results of Schellinger 1951 for the ball milling of silica sand [29]. Interestingly, this value was determined from a comparison between the dissipated thermal energy (obtained by calorimetry) and the mechanical energy consumed by the mill (using a torque sensor). 


\subsection{Agglomeration}

Fig. 7 shows the evolution of the agglomerated surface estimated by using Eq. 6 as a function of the SSA $S$. Below $S=1500 \mathrm{~m}^{2} \cdot \mathrm{kg}^{-1}$, the agglomerated surface is low and constant. A sudden increase of agglomeration is observed when $S$ exceeds $1500 \mathrm{~m}^{2} \cdot \mathrm{kg}^{-1}$. Interestingly, this transition occurs for the same value of $S$ for which the experimental results begin to deviate from the Rittinger model, suggesting that the decrease in grindability mainly reflects the cohesive surface forces and the resulting re-agglomeration of fine particles inside the powder.

Figure 7: Agglomerated surface (specific surface area liberated by ultrasound) as a function of the specific surface area for each specific impact energy $E i_{m}$ in $\mathrm{mJ} / \mathrm{g}$.

At low impact energies, the agglomerates can be visually identified at the end of the milling (Fig. 8a). For higher impact energies $(20 \mathrm{~Hz}, 6 \mathrm{~g}$ and $25 \mathrm{~Hz}, 13 \mathrm{~g}$ ), the agglomeration of particles eventually leads to clogging phenomena as observed in Fig. 8b.

Figure 8: Agglomerates (13 g,20 Hz) (left) and sticking powder on the jar wall $(13 \mathrm{~g}, 25 \mathrm{~Hz})$ (right) at the end of milling.

In order to clarify the effect of the finest particles, we studied the correlation between agglomeration and volume fraction $\theta_{d}$ of particles below a limit size $d=0.5 \mu \mathrm{m}$. Fig. 9 shows $\theta_{d}$ as a function of $S$. As in Fig. 7, the curve is bilinear with a clear change in slope at $S \simeq 1500$ $\mathrm{m}^{2} \cdot \mathrm{kg}^{-1}$. This correlation suggests that the proportion of finest particles is a crucial parameter for the milling efficiency. We observe a similar agglomeration transition for even coarser particles by choosing a slightly larger value of $d$, but the cohesive effects are more clearly expressed for $d$ $=0.5 \mu \mathrm{m}$. Note also that, even if agglomeration occurs all along the milling, its hindering effect on the efficiency occurs only when the volume fraction of particles increases to a critical value, which is actually quite low (around $0.4 \%$ ).

Figure 9: Evolution of the volume fraction $\theta_{0} .5$ of particles of size below $0.5 \mu \mathrm{m}$ as a function of the SSA. 


\subsection{Energy consumption}

In this section, we consider the electric energy consumed by the device and heat loss while milling. Fig. 10 shows, as a function of time, the electric power $P$ and the temperature $T$ measured on the jar during the milling of $20 \mathrm{~g}$ of silica sand at $20 \mathrm{~Hz}$.

For milling times below $72 \mathrm{~min}, P$ decreases and reaches a plateau. Although the origin of this transient is not clear, it may be attributed to a better lubrication of mechanical parts at higher temperatures. During this first phase, the temperature evolves linearly. After 72 min a sudden power drop occurs and $P$ stabilizes to a lower value. In this regime, the temperature decreases exponentially, and eventually levels off.

Figure 10: Evolution of the electric power and temperature of the jar as a function of time for $20 \mathrm{~g}$ $20 \mathrm{~Hz}$.

\begin{tabular}{|c|c|}
\hline Mass (g) & Power drop (\%) \\
\hline 6 & 1.8 \\
\hline 13 & 3.5 \\
\hline 17 & 17.5 \\
\hline 20 & 33.8 \\
\hline
\end{tabular}

Table 1: Percentage of power lost after the transition for a fixed frequency of $20 \mathrm{~Hz}$.

Several experiments were also carried out for other values of the powder mass and vibration frequency. Similar trends were observed but with smaller values of energy power drop, as reported in Table 1 for different filling ratios. For all the experiments, this transition appears for a surface $S \simeq 2990 \mathrm{~m}^{2} \mathrm{~kg}^{-1}$, close to the asymptotic limit of the modified Rittinger model ( $\left.S_{0} \tau / \gamma=3140 \mathrm{~m}^{2} \mathrm{~kg}^{-1}\right)$.

We also measured the effect of powder mass on energy consumption before and after the the transition observed in Fig. 10. Let $P_{1}$ be the electric power consumed before the transition and $P_{2}$ the electric power after the transition. $P_{1}$ mainly contains the power consumed by the device, frictional interactions and particle breakage whereas $P_{2}$ contains also the effects of 
agglomeration. We therefore expect $P_{2}$ to follow the same trend as $P_{1}$ as a function of powder mass $M$ for small values of the latter. To quantify dissipation due to the device, we also measured the electric power $P_{0}$ of the device with a jar containing a fixed weight of mass equal to the total mass of ball and product. Fig. 11 shows $P_{0}, P_{1}$ and $P_{2}$ as a function of $M$. As powder mass is small compared to the mass of the jar, $P_{0}$ does not vary significantly with $M$. As expected, $P_{1}$ increases linearly with $M$, and $P 2$ follows initially $P 1$, but tends to decrease as $M$ further increases. It tends to $P_{0}$, meaning that the ball stroke vanishes at large values of $M$ by particle agglomeration and hence the whole dissipation is reduced to that of the device.

Figure 11: Evolution of the electric power as a function of the powder mass when the mass is fixed ( $P_{0}$, squares $)$, and when it is free before $\left(P_{1}\right.$, circles $)$ and after transition $\left(P_{2}\right.$, triangles $)$.

\section{Conclusions}

In this work, we preformed an extensive parametric study of the milling of silica sand using an oscillatory ball mill. We showed that the extra surface created by milling depends on the total energy supplied, and a dimensionless parameter $\left(E_{m}\right)$, representing the specific grinding energy, is the control parameter of the generated specific surface area and fully mediates the influence of all experimental parameters (frequency, ball stroke) on the grinding process.

For specific surface area above $1500 \mathrm{~m}^{2} \mathrm{~kg}^{-1}$, an increasing discrepancy with the classical Rittinger model was observed and shown to reflect the action of cohesive forces and its agglomerating effect on the class of fine particles. A modified model was shown to account for the agglomeration effect with the prediction of an asymptotic limit value of the SSA. Moreover, it was shown that the agglomeration is strongly correlated with the volume fraction of the smallest particles. The signature of the agglomeration phenomena was clearly identified from the electrical power consumed by the device.

\section{Acknowledgements}

We thank Bpifrance for their financial support (ACA project). 


\section{References}

\section{References}

[1] D. Touil, S. Belaadi, C. Frances, The specific selection function effect on clinker grinding efficiency in a dry batch ball mill, International Journal of Mineral Processing 87 (3-4) (2008) 141-145. doi:10.1016/j.minpro.2008.02.003.

[2] T. Tanaka, A new concept applying a final fineness value to grinding mechanism-grinding tests with frictional and impulsive force, Kagaku Kogaku 18 (1954) 160-171.

[3] L. Tavares, R. King, Single-particle fracture under impact loading, International Journal of Mineral Processing (1998). doi:10.1016/S0301-7516(98)00005-2.

[4] P. R. Rittinger, Lehrbuch der Aufbereitungskunde, Ernst and Korn, Berlin, 1867.

[5] F. C. Bond, The third theory of comminution, Transactions on AIME Mining Engineering 193 (1952) 484-494.

[6] F. Kick, Das Gesetz des proportionalen Widerstands und seine Anwendug, Leipzig, F. (Ed.), 1885.

[7] G. Mucsi, Grindability of quartz in stirred media mill, Particulate Science and Technology 31 (4) (2013) 399-406. doi:10 .1080/02726351.2013.767294.

[8] Y. Chen, Y. Ding, D. G. Papadopoulos, M. Ghadiri, Energy-Based Analysis of Milling -Lactose Monohydrate, Journal of Pharmaceutical Sciences 93 (4) (2004) 886-895.

[9] P. Karinkanta, M. Illikainen, J. Niinimäki, Impact-based pulverisation of dried and screened Norway spruce (Picea abies) sawdust in an oscillatory ball mill, Powder Technology 233 (2013) 286-294. doi:10 .1016/j . powtec.2012 .09.011. URL http://dx.doi.org/10.1016/j.powtec.2012.09.011

[10] H. Cho, M. A. Waters, R. Hogg, Investigation of the grind limit in stirred-media milling, International Journal of Mineral Processing 44-45 (SPEC. ISS.) (1996) 607-615. doi:10.1016/0301-7516(95)00069-0.

[11] P. L. Guzzo, A. A. Tino, J. B. Santos, The onset of particle agglomeration during the dry ultrafine grinding of limestone in a planetary ball mill, Powder technology 284 (2015) $122-129$.

[12] V. V. Boldyrev, S. V. Pavlov, E. L. Goldberg, Interrelation between fine grinding and 
mechanical activation, International Journal of Mineral Processing 44-45 (SPEC. ISS.) (1996) 181-185. doi:10.1016/0301-7516(95)00028-3.

[13] K. Kendall, The impossibility of comminuting small particles by compression (1978). doi:10.1038/279169d0.

[14] C. E. Renshaw, E. M. Schulson, Universal behaviour in compressive failure of brittle materials, Nature 412 (August) (2001) 897-900.

[15] L. G. Austin, P. Bagga, An analysis of fine dry grinding in ball mills, Powder Technology 28 (1) (1981) 83-90. doi : $10.1016 / 0032-5910$ (81) 87014-3.

[16] J. Kano, F. Saito, Correlation of powder characteristics of talc during planetary ball milling with the impact energy of the balls simulated by the particle element method, Powder Technology 98 (2) (1998) 166-170. doi:10.1016/S0032-5910 (98)00039-4.

[17] E. Teke, M. Yekeler, U. Ulusoy, M. Canbazoglu, Kinetics of dry grinding of industrial minerals: Calcite and barite, International Journal of Mineral Processing 67 (1-4) (2002) 29-42. doi:10.1016/S0301-7516(02)00006-6.

[18] N. Kotake, M. Kuboki, S. Kiya, Y. Kanda, Influence of dry and wet grinding conditions on fineness and shape of particle size distribution of product in a ball mill, Advanced Powder Technology 22 (1) (2011) 86-92. doi:10.1016/j.apt.2010.03.015.

URL http://dx.doi.org/10.1016/j.apt.2010.03.015

[19] W. Choi, H. Chung, B. Yoon, S. Kim, Applications of grinding kinetics analysis to fine grinding characteristics of some inorganic materials using a composite grinding media by planetary ball mill, Powder technology 115 (3) (2001) 209-214.

[20] H. Choi, W. Lee, S. Kim, Effect of grinding aids on the kinetics of fine grinding energy consumed of calcite powders by a stirred ball mill, Advanced Powder Technology 20 (4) (2009) 350-354.

[21] P. A. Webb, A primer on particle sizing by static laser light scattering, Technical Workshop Series: Introduction to the Latest ANSI/ISO Standard for Laser Technical Workshop Series: Introduction to the Latest ANSI/ISO Standard for Laser Particle Size Analysis (2000).

[22] G. R. McDowell, M. D. Bolton, On the micromechanics of crushable aggregates, Géotechnique 48 (5) (1998) 667-679. doi:10.1680/geot.1998.48.5.667. URL http://dx.doi.org/10.1680/geot.1998.48.5.667 
[23] H. J. Herrmann, S. Roux, Statistical models for the fracture of disordered media, Elsevier, 1990.

[24] H. Laubie, S. Monfared, F. Radja, R. Pellenq, F.-J. Ulm, Effective potentials and elastic properties in the lattice-element method: Isotropy and transverse isotropy, Journal of Nanomechanics and Micromechanics 7 (3) (2017) 04017007.

[25] J. T. Hagan, Micromechanics of crack nucleation during indentations, Journal of Materials Science 14 (12) (1979) 2975-2980. doi : 10.1007 /BF0 0611482.

[26] L. Opoczky, Fine grinding and agglomeration of silicates, Powder Technology 17 (1) (1977) 1-7.

[27] J. Seville, C. Willett, P. Knight, Interparticle forces in fluidisation: a review, Powder Technology 113 (3) (2000) $261-268$.

doi:https://doi.org/10.1016/S0032-5910(00)00309-0.

URL

http://www.sciencedirect.com/science/article/pii/s003259100 0003090

[28] G. Nichols, S. Byard, M. J. Bloxham, J. Botterill, N. J. Dawson, A. Dennis, V. Diart, N. C. North, J. D. Sherwood, A review of the terms agglomerate and aggregate with a recommendation for nomenclature used in powder and particle characterization, Journal of pharmaceutical sciences 91 (10) (2002) 2103-2109.

[29] D. W. Fuerstenau, A. Z. Abouzeid, The energy efficiency of ball milling in comminution, International Journal of Mineral Processing 67 (1-4) (2002) 161-185.

doi:10.1016/S0301-7516(02)00039-X. 